\title{
COMUNICAÇÃO E CULTURA ORGANIZACIONAL EM CONTEXTOS DE TRANSIÇÃO TECNOLÓGICA
}

\author{
Communication and organizational culture in the \\ contexts of technology transition
}

Renato Dias Baptista ${ }^{1}$

\section{Resumo}

As novas tecnologias redesenham o cenário organizacional e impactam sobre a cultura e os sistemas de trabalho. Atreladas a essas transformações, estão a globalização e as imposições da velocidade dos métodos produtivos, da pressão pela renovação contínua e da rápida obsolescência. Nesse cenário, a comunicação possibilita um redesenho dos processos evolutivos das empresas e passa a ocupar um espaço de elevada importância para a elaboração de estratégias organizacionais.

Palavras-chave: Comunicação; Cultura; Novas tecnologias; Comportamento humano.

\section{Abstract}

The new technologies redesign the organizational scene and impact on the culture and the systems of work. Linked to these transformations are the globalization and the speed impositions of the productive methods, the pressure for the continuous renewal and of the fast obsolescence. In this scene, the communication makes the possible redesigns of the evolutions processes of the companies starts to occupy a greater importance an elaboration of strategic organization.

Keywords: Communication; Culture; New technologies; Human behavior.

\section{Introdução}

Somos capazes de resistir às informações que não adaptam à nossa ideologia, percebendo essas informações não como informações, mas como trapaças ou mentiras.

Edgar Morin, Para sair do século XX

\footnotetext{
Doutorando em Comunicação e Semiótica pela Pontifícia Universidade Católica de São Paulo (PUC/SP), Mestre em Comunicação (UNESP). Atualmente é docente da Universidade Paulista e das Faculdades Integradas de Bauru nas disciplinas de Psicologia da Comunicação e Comportamento Humano nas Organizações. e-mail: rdbapt@gmail.com
} 
A evolução tecnológica fomenta intensas transformações organizacionais que provocam impactos sobre os valores humanos. As interações sociais, as concepções sobre o trabalho, o lazer, entre outros, são arremessados num turbilhão movimentado pela globalização e apoiados por robôs, sistemas especialistas, tecnologias da informação e técnicas de gestão.

Nesse cenário, a comunicação organizacional redescobriu seu papel, mas como todo conhecimento, pode ser um instrumento de esclarecimento ou manipulação. $O$ fator esclarecimento está associado com outros métodos éticos e socialmente responsáveis que se vinculam às mudanças. $\mathrm{Na}$ outra extremidade, quando a comunicação é manipulativa, ela alimenta as novas formas de tortura no trabalho; em ambientes tirânicos, ela é concebida como uma mera "ferramenta" para o controle organizacional, além de amparar discursos vinculados à lógica da autoexclusão. Há programas corporativos que apresentam a tese de que não seriam as empresas que excluem, a exclusão é causada pelos próprios empregados que não se adaptam ao ritmo acelerado das mudanças.

A propósito, esse é um dos maiores impactos contemporâneos; a imposição de velocidade aos métodos de trabalho. Esse deslocamento está presente nos sistemas produtivos, nas estratégias e na informação instantânea. Não há sintonia com as subjetividades num contexto em que as tecnologias incitam a reordenação e desprezam as culturas (BAPTISTA, 2006a).

As organizações criaram a tortura do imediatismo; uma ardileza do conceito de flexibilização empresarial, um recurso subserviente ao mercado global.

Outras conseqüências dessas mudanças direcionam-se aos estreitamentos no ciclo de aprendizagem e desenvolvimento cognitivo, há uma contraposição aos métodos da assimilação tradicional de conceitos. Todos devem aprender rapidamente e, na mesma velocidade, renunciarem ao passado. Rompem-se os conhecimentos que são considerados, rapidamente, obsoletos na sociedade contemporânea.

Diante desses pressupostos, o objetivo principal deste artigo é apresentar uma visão panorâmica sobre os contextos organizacionais contemporâneos e as interdependências com a comunicação e a cultura. Para essa consecução, buscamos os pressupostos e pesquisas de autores como Adler (2002); Antunes (2000); Aubert (2003); Baptista (1997, 2006b, 2007); Bueno (2005); Chesneaux (1996); Trivinho (2001); Walton (1998); entre outros, para caracterizar essa tríplice influência.

\section{Comunicação, cultura e tecnologia}

O intenso redesenho tecnológico gera impactos na cultura organizacional que, por sua vez, requerem estratégias comunicacionais para amenizar rejeições às mudanças. Há um aspecto fundamental nesse processo: a comunicação deve ser ética. Ela é mais do que uma simples informação ou um instrumento de controle e disciplina; necessita associar-se às transformações gradativas, respeitar os múltiplos clientes e vincular-se à Responsabilidade Social.

Entendemos que as empresas visam ao lucro, portanto, a comunicação não será um mecanismo de benevolência, mas é capaz de garantir, junto às ações de gestão de pessoas e planejamento macroestratégico, a continuidade de uma empresa e o apreço em direção de uma evolução no ambiente de trabalho. Assim, consideramos que uma organização avança quando ela gera pessoas de forma inteligente, associa seus colaboradores com as tecnologias, investe em treinamento contínuo, abre os canais comunicacionais de forma efetiva e busca os pressupostos da participação no trabalho.

A importância da comunicação e cultura pode ser observada em todos os processos de aquisição tecnológica. Uma pesquisa realizada em empresas no Estado de São Paulo possibilitou a comprovação dessa tríplice dependência. Nas ações de reestruturação de tecnologias ocorrida nos finais dos anos 90, foram encontrados dois procedimentos em diferentes companhias: no primeiro, caracterizado como desenvolvimento contínuo, havia uma preocupação em relação à capacitação ininterrupta dos recursos humanos, nesse caso, possibilitava-se que os trabalhadores estivessem cognitivamente preparados para transições tecnológicas. No segundo, constituído de empresas mais lentas, as "estratégias" mais comuns eram as imposições para a mudança e a não observância da cultura estabelecida (BAPTISTA, 1997, 2007).

Em relação às empresas que respeitaram os valores, havia um estudo de clima e dos pressupostos culturais antes de se proceder as 
mudanças. Com o apoio de dados sobre essas variáveis, tornaram-se estrategistas, éticas e responsáveis socialmente. Nesse tipo de ambiente, há uma absorção gradativa que garante a efetiva transformação. Em situação oposta, aquelas que utilizaram a mudança autoritária foram morosas e demandaram maiores investimentos, pois seus métodos requeriam um rígido controle que, a propósito, destruíam as relações humanas.

A rigidez na mudança pode fomentar conflitos que estimulam as resistências comportamentais. Baptista (2006c) cita, como exemplo, uma empresa que apregoava o slogan "nossos trabalhadores são o nosso principal patrimônio", porém, o local estava em alto nível de pressão e essa frase falaciosa ocasionava, na verdade, muitas dissonâncias contraproducentes.

Como a tentativa de exercer controle autoritário nem sempre garante o êxito, a percepção de engodo que os funcionários possuem pode comprometer a veracidade dos planos futuros. Concordo com Adler (2002) em relação à idéia de que, ao fazermos suposições sobre o que percebemos, tendemos em não redescobrir significados cada vez que encontramos uma situação similar. Nesse aspecto, o erro ou a falácia tornam "infecundo" o ambiente; a equipe amplia suas concepções aversivas e tornam-se poucos predispostos às estratégias comunicacionais sobre as necessárias mudanças.

\section{Uma gestão competente é capaz de gerir a verdade}

Para efetivamente gerir esse contexto, é essencial assimilar que há uma categórica confluência da comunicação com a cultura organizacional; ambas inter-relacionam-se devido às características sistêmicas (LITTLEJOHN, 1988), orgânicas (MATTELART, 1994) e de processos, mas é com o desenvolvimento da microinformática e da automação que a cultura arraigou-se como base para a transição empresarial.

Mas onde está a cultura? Inicialmente, devemos buscar os conceitos fundamentais para dissecar essa complexidade. Para Hofstede (2004), dentre os muitos termos usados para descrever as manifestações da cultura, podemos reter quatro deles que cobrem o conceito de forma minuciosa: símbolos, heróis, rituais e valores. Para o autor, os símbolos são palavras, gestos, figuras ou objetos que transportam um significado particular que é apenas reconhecido pelos que partilham a cultura. Os heróis são pessoas que possuem características altamente valorizadas numa determinada cultura e que por isso servem de modelo de comportamento. Os rituais são atividades coletivas, tecnicamente supérfluas, para atingir fins desejados, mas considerados essenciais em determinada cultura; formas de cumprimentar ou transmitir respeito aos outros constituem alguns exemplos.

Conforme Deal e Kennedy (1992), os valores, por sua vez, são a essência de uma filosofia da empresa para alcançar o sucesso, eles provêem um senso de direção comum para todos os empregados e as diretrizes para o comportamento do dia-a-dia deles. São fórmulas que determinam (e ocasionalmente resultam disso) os tipos de heróis corporativos, os mitos, os rituais e as cerimônias da cultura.

Valendo-nos dos pressupostos Adler (2002) e Hofstede (2004), destacamos que as diferentes manifestações da cultura são decodificadas, assimiladas, questionadas e absorvidas mediante estratégias de gestão da imagem corporativa, a integração ao trabalho, as regras e suas formas de implementação, o fluxo de tarefas, a acessibilidade entre a base e o topo da hierarquia, os veículos de informação interna e suas formas de linguagem, ou seja, comunicação.

Portanto, são os sistemas comunicacionais que permitem que os funcionários percebam as dissonâncias, que, por sua vez, deterioram as relações com a empresa. O que se vê pode refutar aquilo que se diz e resultar em desmotivação, ansiedade e conflitos intergrupais (BAPTISTA, 2006c).

Conforme Bueno (2005, p. 24):

É preciso mudar o perfil da comunicação interna para que ela efetivamente assuma o seu caráter libertador. Isso não significa tornar mais eficazes os canais para a consolidação da hegemonia das chefias, como preconiza a literatura comprometida com a velha ideologia institucional, mas criar espaços de interação democráticos, verdadeiramente participativos, onde a divergência, com responsabilidade, seja assumida.

Diante desse conjunto de elementos, se as organizações não desenvolverem sistemas consonantes com os valores humanos, promoverão as novas torturas do trabalho caracterizadas pela velocidade imperativa. 
Vivemos num mundo caracterizado por uma ditadura do tempo real, pela sociedade do zapping e dos clips nos quais vivem intensamente sem duração na busca de resultados de eficácia imediata (AUBERT, 2003). É uma corrida do mundo fugaz. (CHESNEAUX, 1996), uma obsessão pela automatização e pela racionalização organizacional (LACOSTE, 2005).

O indivíduo "deve" absorver as idéias de uma organização que gera ansiedade através da velocidade de trabalho mensurada pelas oscilações do mercado. No entendimento de Aubert (2003), vivemos de ações mais imediatas numa aliança que se operou entre a lógica do lucro e os métodos de gerenciamento associados à urgência.

Essas confluências de impactos tecnológicos levam Trivinho (2001) a afirmar que:

Os novos códigos se caracterizam, sociologicamente, como dispositivos tecnoculturais de acesso à práxis instrumental na sociedade tecnológica. Em outras palavras, como uma senha geral de permissões, de direitos enfim, essas linguagens funcionam como gerenciadores da entrada tanto no mercado de trabalho, quanto na vivência dos demais processos, como, por exemplo, a fruição dos novos bens culturais, que já proliferam de modo ostensivo, igualmente sem uma avaliação satisfatória por parte da reflexão teórica. Isso representa, no fundo, o acesso a uma nova simbólica, ou melhor, a um novo mundo tecnosimbólico, que envolve uma tecnoaculturação específica do psiquismo humano, correspondente à era da informatização. (TRIVINHO, 2001, p. 91).

Nessas circunstâncias, ressalta Chesneaux (1996), todos cedem à pressão estressante do imediato e somos agarrados pelo efêmero. A pressão da informação transforma a vida cotidiana em antecipação angustiada do dia seguinte, perdendo cada dia sua realidade viva e específica. "As novas tecnologias se propõem a aumentar quase ao infinito nossas capacidades de produzir e de reproduzir, de gerar a paz e fazer guerra, de divertir-se ou de pensar" (CHESNEAUX, 1996, p. 110).

$\mathrm{Na}$ opinião de Chesnais (1996), a convergência das tecnologias de informática e de telecomunicações, bem como a introdução de tecnologias ligadas às comunicações por satélite, ao comando numérico e das fibras óticas criaram condições para o estabelecimento de um sistema verdadeiramente mundial. Ainda segundo o mesmo autor, as transformações advindas, desde fins da década de 70, nas relações entre ciência, tecnologia e atividade industrial, fizeram da tecnologia um fator de competitividade, cujas características afetam praticamente todo o sistema.

\section{Homem-máquina no cenário organizacional}

Há inúmeros elementos que engendram os domínios das máquinas num contexto que, de acordo com Walton (1998), engloba robôs, movimentação de materiais, engenharia e planejamento de processos assistidos por computador, planejamento e controle e gestão de sistemas de suporte e decisão.

No pretexto da sobrevivência num mercado global, valorizam-se as qualidades daqueles capazes de se adequarem aos preceitos atuais. As tecnologias redefinem as concepções nas organizações e contrapõem o cenário iniciado com a primeira Revolução Industrial. O termo revolução, a propósito, traz consigo a essencial revisão daquilo que é vigente e estimula a geração de prognósticos, nem sempre pertinentes, sobre o futuro do trabalho. Landes (1998) ressalta que a palavra revolução pode evocar visões de mudança rápida, até brutal ou violenta. Pode significar também transformação fundamental ou profunda. Para alguns, destaca o autor, tem conotações progressistas, para outros as revoluções são intrinsecamente destrutivas. Todos esses e outros significados dependem de uma palavra que outrora significava simplesmente uma rotação no sentido literal.

Um breve olhar sobre a história mostra que as primeiras máquinas que alavancaram as fábricas do passado apresentaram estágios de evolução técnica mais longa em comparação aos fundamentados na microeletrônica. A distância entre um invento e outro favoreceu amplas adequações dentro e fora das organizações.

Segundo Landes (1998), o primeiro engenho a usar vapor para criar um vácuo e fazer funcionar uma bomba foi patenteado na Inglaterra por Thomas Savery, em 1698; a primeira máquina a vapor propriamente dita (com pistão) foi a de Thomas Newcomen, em 1705. Um longo período transcorreu antes de James Watt inventar uma máquina com condensador separado do cilindro (1768), cuja eficiência era suficientemente boa para produzir vapor fora das minas, nas novas cidades 
industriais; e mais de 15 anos foram necessários para adaptar a máquina ao movimento rotativo, de modo a poder impulsionar as rodas da indústria.

Esses fatos caracterizaram a primeira revolução, situada entre o final do século XVII e o início do século XIX que, conforme Schaff (1996), teve o grande mérito de substituir na produção a força física do homem pela energia das máquinas; primeiro pela utilização do vapor e mais adiante pela utilização da eletricidade.

Concordo com Vonnegut Jr. (1999) quando diz que estamos em meio ao processo de desvalorizar o pensamento humano, pois o ritmo das máquinas fundamentadas na microeletrônica e seus conseqüentes impactos nas técnicas de trabalho não permitem espaços adequados de absorção e recomposição de novas subjetividades (BAPTISTA, 2006a). "A analogia com a primeira revolução está no salto qualitativo operado no desenvolvimento da tecnologia de produção que acabou por romper a continuidade dos avanços quantitativos que se iam acumulando nas tecnologias já existentes" (SCHAFF, 1996, p. 22).

Mas as empresas não são ambientes de quietude, a despeito de todo o discurso de valorização humana, o lucro não cede espaço. A história, segundo Womack et al. (1992), também revela que as primeiras modificações desenvolvidas por Ford no processo de trabalho não se limitaram a aperfeiçoar a peça intercambiável, mas também aperfeiçoaram o operário intercambiável.

A estratégia, mais dinheiro com menos pessoas, prosseguir-se-á nas novas etapas dos sistemas produtivos em que se implantam tecnologias com um ímpeto que causa rupturas nas tradicionais formas do desenvolvimento das tarefas. Para Chesneaux (1996), nem a máquina a vapor, nem o telefone, nem a eletricidade, muitas vezes invocados para celebrar a continuidade segura do progresso técnico, provocaram, na sua época, tal choque visceral e difuso.

Diante de tal situação, a classe que vive do trabalho sofre uma aguda crise que atinge não só a sua materialidade, mas tem profundas repercussões na sua subjetividade e, no íntimo interrelacionamento destes níveis, afetou a sua forma de ser (ANTUNES, 2000). O computador, por exemplo, é evidenciado por Chesneaux (1996) como algo que surge, contando em pico-segundos e nanosegundos, na plenitude do controle racional dos ritmos e das seqüências de trabalho que aspiraram ao fordismo e ao taylorismo.
Assim, essas tecnologias impelem a reordenação, extrapolam espaços que antes eram considerados privados, controlam o tempo de lazer, criam o trabalho portátil, cingem os que são tragados e incitam a culpa de forma unilateral aos que são excluídos (BAPTISTA, 2006a).

Nesse novo darwinismo (KURZ, 1999), é preciso absorver tudo de forma rápida além de, conforme Lacoste (2005), exigir a mobilização de conhecimento e saberes múltiplos; os recursos cognitivos são condições essenciais para o êxito.

\section{Considerações finais}

A comunicação e a cultura devem ser repensadas num ambiente de alta tecnologia. É necessário redescobrir seu papel diante da intensa velocidade. Vivemos num sistema de mútuas interferências, mas que não auto-regula-se com a mesma rapidez da tecnologia; para efeito de evolução, nenhuma sociedade ou empresa poderia esperar por uma auto-regulação.

É fato que as novas tecnologias formam a "paisagem corporativa" e, a despeito de todos os problemas, não haverá retrocesso. Diante disso, o estudo dos impactos humanos que estão associados nesse processo não deve funcionar como espasmos do pensamento, como a crítica pela crítica, como deleite intelectual.

Por fim, a integralidade do sistema comunicacional deve ser efetiva; afastar-se do discurso, da dissonância, da manipulação, da subserviência às empresas ausentes de Responsabilidade Social. Uma comunicação ética é possível, primeiramente, é preciso associá-la à cultura e à gestão competente de pessoas.

\section{Referências}

\section{ADLER, J. A. International dimensions of organizational behavior. Toronto: South- Western, 2002.}

ANTUNES, R. Adeus ao trabalho? Ensaio sobre as metamorfoses e a centralidade do mundo do trabalho. Campinas: UNICAMP; São Paulo: Cortez, 2000.

AUBERT, N. Le culte de 1'urgence. Paris: Flamarion, 2003. 
BAPTISTA, R. D. O processo de comunicação e clima organizacional na entrada de novas tecnologias. 1997. 157 f. Dissertação (Mestrado em Comunicação) - Faculdade de Arquitetura, Artes e Comunicação da Universidade Estadual Paulista, São Paulo, 1997.

Será que a estagnação é um mecanismo de absorção e de recomposição da subjetividade? Disponível em: <http:/ / netart.incubadora.fapesp.br/ portal/Members/rdbap/guattari>. Acesso em: 31 ago. $2006 a$.

Comunicação e cultura organizacional: interfaces com as novas tecnologias. In: ENCONTRO DA UNIÃO LATINOAMERICANA DE ECONOMIA POLÍTICA DA INFORMAÇÃO, DA COMUNICAÇÃO E DA CULTURA. 2006, Niterói. Anais... Niterói: Universidade Federal Fluminense, 2006b.

- Trabalho e transitoriedade tecnológica: as compressões da mudança num contexto de globalização. In: ENCONTRO DE PÓSGRADUAÇÃO EM PSICOLOGIA, 2006, Assis. Disponível em: <http://www.assis.unesp.br/ encontrosdepsicologia $>$. Acesso em: 30 ago. 2006c.

. As dimensões da comunicação e da cultura organizacional em um contexto de renovação tecnológica. Revista Comunicação e Sociedade, São Bernardo do Campo, n. 46, p. 33-41, 2006d.

Involuções Corporativas: perspectivas críticas sobre a gestão de pessoas, comunicação e cultura nas organizações. São Paulo: All Print, 2007.

BUENO, W. C. Comunicação empresarial no Brasil: uma leitura crítica. São Paulo: All Print, 2005.

CHESNAIS, F. A mundialização do capital. São Paulo: Xamã, 1996.

CHESNEAUX, J. Modernidade-mundo. Petrópolis: Vozes, 1996.

DEAL, T. E; KENNEDY, A. A. Corporate cultures: the rites and rituals of corporate life. New York: Addison-Wesley, 1992.
HOFSTEDE, G. Cultures and organizations: software for the mind. New York: McGraw-Hill, 2004.

KURZ, R. O colapso da modernização: da derrocada do socialismo de caserna à crise da economia mundial. Rio de Janeiro: Paz e Terra, 1999.

LANDES, D. S. A riqueza e a pobreza das nações: por que algumas são tão ricas e outras tão pobres. Rio de Janeiro: Campus, 1998.

LACOSTE, M. Peut-on travailler san communiquer? In: Borzeix, A.; Fraenkel, B. (Coord.). Langage et travail: communication, cognition, action. Paris: CNRS, 2005.

LITTLEJOHN, S. W. Fundamentos teóricos da comunicação humana. Rio de Janeiro: Guanabara, 1988.

MORIN, E. Para sair do século XX. Rio de Janeiro: Nova Fronteira, 1986.

MATTELART, A., MATTELART, M. A invenção da comunicação. Lisboa: Instituto Piaget, 1994.

SCHAFF, A. A sociedade informática. São Paulo: UNESP; Brasiliense, 1996.

TRIVINHO, E. O mal-estar da teoria: a condição crítica na sociedade tecnológica atual. Rio de Janeiro: Quartet, 2001.

VONNEGUT Jr., K. Player piano. New York: Dial Press, 1999.

WALTON, R. E. Tecnologia de informação: o uso de TI pelas empresas que obtêm vantagem competitiva. São Paulo: Atlas, 1998.

WOMACK, J. P. et al. A máquina que mudou o mundo. Rio de Janeiro: Campus, 1992.

Recebido em: 14/02/2007 Received in: 02/14/2007 Aceito em: 11/04/2007 Accepted in: 04/11/2007 